\title{
Twiddler's syndrome in spinal cord stimulation
}

\author{
Rafid Al-Mahfoudh $^{1,2} \cdot$ Yuen Chan ${ }^{1} \cdot$ Hsu Pheen Chong ${ }^{1} \cdot$ Jibril Osman Farah $^{1}$
}

Received: 9 June 2015 / Accepted: 28 October 2015 / Published online: 17 November 2015

(C) The Author(s) 2015. This article is published with open access at Springerlink.com

\begin{abstract}
Background The aims are to present a case series of Twiddler's syndrome in spinal cord stimulators with analysis of the possible mechanism of this syndrome and discuss how this phenomenon can be prevented.

Method Data were collected retrospectively between 2007 and 2013 for all patients presenting with failure of spinal cord stimulators. The diagnostic criterion for Twiddler's syndrome is radiological evidence of twisting of wires in the presence of failure of spinal cord stimulation.

Results Our unit implants on average 110 spinal cord stimulators a year. Over the 5-year study period, all consecutive cases of spinal cord stimulation failure were studied. Three patients with Twiddler's syndrome were identified. Presentation ranged from 4 to 228 weeks after implantation. Imaging revealed repeated rotations and twisting of the wires of the spinal cord stimulators leading to hardware failure.

Conclusions To the best of our knowledge this is the first reported series of Twiddler's syndrome with implantable pulse generators (IPGs) for spinal cord stimulation. Hardware failure is not uncommon in spinal cord stimulation. Awareness and identification of Twiddler's syndrome may help prevent its occurrence and further revisions. This may be achieved by implanting the IPG in the lumbar region subcutaneously above the belt line. Psychological intervention may have a
\end{abstract}

Rafid Al-Mahfoudh

rafid@doctors.org.uk; rafidalmahfoudh@yahoo.com

1 The Walton Centre for Neurology \& Neurosurgery, Lower Lane, Liverpool L9 7LJ, UK

2 South East Neurosurgery and Spinal Surgery, Brighton and Sussex University Hospitals NHS Trust, Brighton, UK preventative role for those who are deemed at high risk of Twiddler's syndrome from initial psychological screening.

Keywords Twidder's syndrome $\cdot$ Spinal cord stimulation · IPG $\cdot$ Failed back surgery syndrome

\section{Introduction}

Twiddler's syndrome is rare clinical condition. It is commonly been reported in cardiac pacemakers and implantable cardioverter defribrillators $[5,7,12,13,19,24,27,28,30$, $32,39]$. In neurosurgical practice, Twiddler's syndrome has been reported in deep brain stimulation but never in spinal cord stimulation (SCS) [3, 9, 16, 17, 21, 31, 34]. To the best of our knowledge, we present the first reported case series of hardware malfunction due to Twiddler's syndrome in SCS. Bayliss et al. [5] were the first to report this phenomenon in cardiac pacemakers in 1968. In their case report, it was found that the rotation of the wires was a result of twiddling by the patient. Twiddler's syndrome can be a conscious or subconscious manipulation of an implantable pulse generator (IPG) within its subcutaneous pocket. This ultimately leads to hardware failure, which is often the mode of presentation. There are many components of an SCS and each providing a possible point of failure. Hardware failure can be due to breakage, infection, migration [36]. In Twiddler's syndrome in addition to the radiological evidence, there is raised impedance leading to system failure, loss of capture and a recurrence of symptoms.

\section{Materials and methods}

An average of 110 spinal cord stimulators are inserted per year in our unit. All patients presenting with failure of spinal cord 
stimulators between 2007 and 2013 were reviewed to identify those with evidence of Twiddler's syndrome. Three patients with radiological evidence of Twiddler's syndrome were identified. Informed consent was obtained from these three patients to be included in this study. Radiological evidence consisted of twisting and rotation of the wires. Their case notes, images and management were reviewed. All patients underwent a pain management program (PMP) and psychological assessment prior to considering implanting an SCS. Our department's policy is to obtain routine postoperative radiological images for all cases of SCS as a baseline. In cases of clinical failure of the device, investigations include interrogating the device and radiological imaging to exclude disconnections, lead breaks and other causes of hardware failure.

\section{Results}

\section{Case report 1}

A 54-year-old woman with failed back surgery syndrome following a lumbar microdiscectomy had a spinal cord stimulator inserted in October 2009. A Specify 5-6-5 electrode (Medtronic, Minneapolis, MN, USA) was implanted with the connectors tunnelled subcutaneously and connected to the IPG in the right iliac fossa. There were no intraoperative or postoperative complications. The patient experienced good relief of lower limb pain with stimulation.

At the point of insertion, she weighed $78.8 \mathrm{~kg}$, with a height of $157 \mathrm{~cm}$ (body mass index [BMI], 31.9). Her prePMP assessment revealed moderate levels of depression and high levels of pain-related disability. Her Beck Depression Inventory score was 18 (mild). She had a Pain Anxiety Symptoms Scale of 60 (mean, 94; SD, 39; range 0-200, where $0=$ no pain anxiety and $200=$ severe pain anxiety).

In October 2011, she underwent reprogramming with failure to achieve satisfactory stimulation. Her imaging revealed twisting of the connecting wires adjacent to the pulse generator (Fig. 1). As it was still partially functioning, she chose to defer revision surgery. Eventually the device failed completely. In May 2012, her IPG was repositioned with new connector leads. The connector wires were found knotted and twisted intraoperatively. The new wires were tunnelled to the right lumbar region above the belt line. No further problems have been reported on follow-up.

\section{Case report 2}

A 31-year-old woman with failed back surgery syndrome following a right lumbar microdiscectomy. Her weight was $93.1 \mathrm{~kg}$ and height $165 \mathrm{~cm}$ (BMI, 34.2). In her pre-PMP assessment, she had moderate depression, moderate pain-related disability and average scores on pain distress and intensity. A

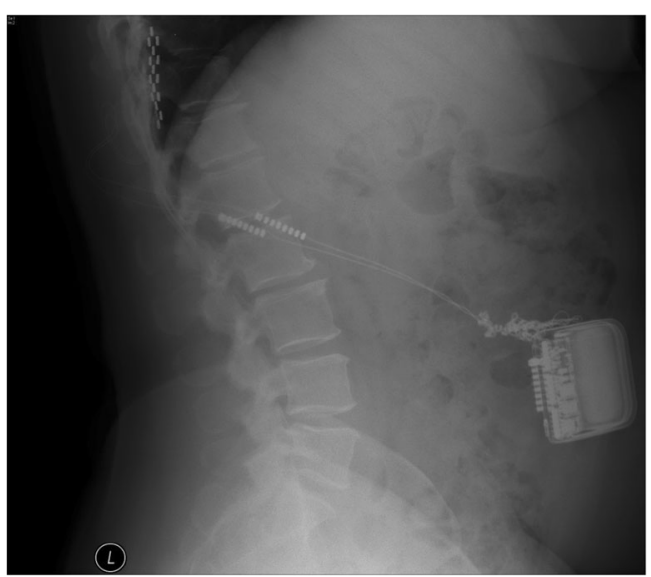

Fig. 1 Lateral plain abdominal X-ray showing twisting of the connecting wires adjacent to the IPG

percutaneous trial of spinal cord stimulation was judged positive and therefore the patient opted for permanent spinal cord stimulator insertion. An eight-electrode surgical lead was implanted with the IPG in the iliac fossa. Postoperatively there was good reduction in pain scores, analgesic usage and mobility; plain X-ray indicated satisfactory electrode placement.

Four weeks after insertion, symptoms recurred to preoperative levels. Interrogation of the system revealed high impedances suggestive of electrode failure. X-ray of the system indicated an abnormal twisting of the wiring between the implanted abdominal pulse generator and the spinal electrode. The subcutaneous pulse generator had undergone repeated rotation, twisting the connecting leads to the point of fracture (Fig. 2).

Surgical revision of the system was performed. The X-ray findings were confirmed intraoperatively. The IPG had rotated on its axis many times (Fig. 3). A new pulse generator was implanted in her right lumbar region above the iliac crest and belt line. Postoperatively, the patient reported restoration of good pain relief.

\section{Case 3}

A 50-year-old woman who initially presented with cauda equina syndrome secondary to a L5/S1 disc prolapse was later diagnosed with failed back surgery syndrome. She weighed $96.95 \mathrm{~kg}$, with a height of 1.57 (BMI, 39.3). This lady had a pain anxiety and symptom scale score of 152 (mean, 94; SD, 39; range, 0-200). On the Becks depression scale, she scored 48 (severe depression).

She had a trial of spinal cord stimulation in 2008, which achieved good control of her pain symptoms. She proceeded to have a spinal cord stimulator (5-6-5 electrode) inserted permanently at the level of T10-12 with connectors tunnelled to the right iliac fossa. 


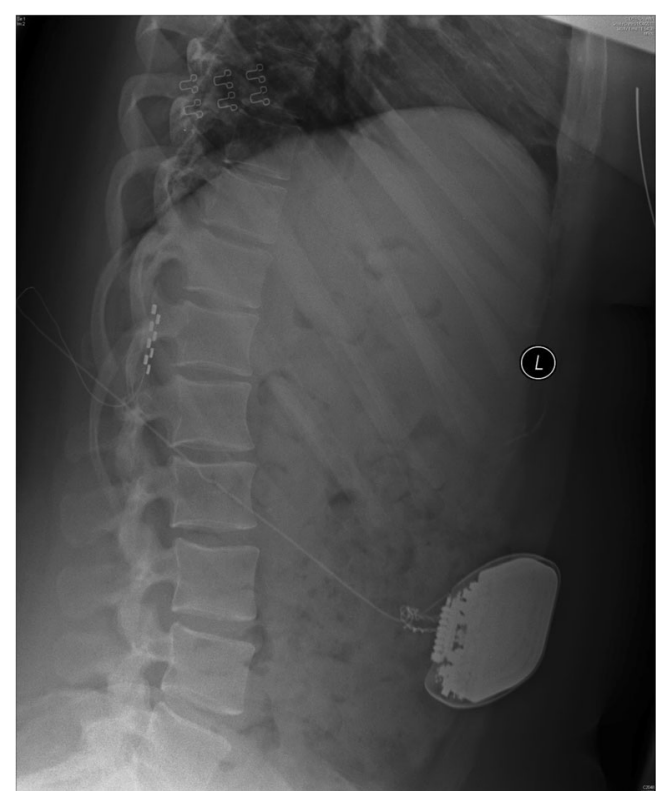

Fig. 2 Lateral plain abdominal X-ray showing multiple twists of the connecting wires adjacent to the IPG

She had good control of her back pain initially; however, during follow-up her pain control deteriorated. In late 2009, she complained of her IPG being mobile and catching on her clothes and it was re-sited at that point. In 2013, she presented with SCS failure. X-rays showed signs of Twiddler's syndrome. On revision of her SCS, the whole extension wires were badly twisted and damaged. She had a replacement extension set tunnelled and connected to the main electrodes, with the IPG repositioned to the lumbar region.

In all three cases the surgical technique included anchoring the lead with the included manufacturer anchoring system. This was by suturing the anchoring system to the lumbar fascia. It was not our policy to anchor the pulse generator in its subcutaneous pocket in the primary surgical procedure. All three patients denied manipulating the IPG. In the revision procedure in all three cases, the IPG was implanted in the lumbar region (Fig. 4).

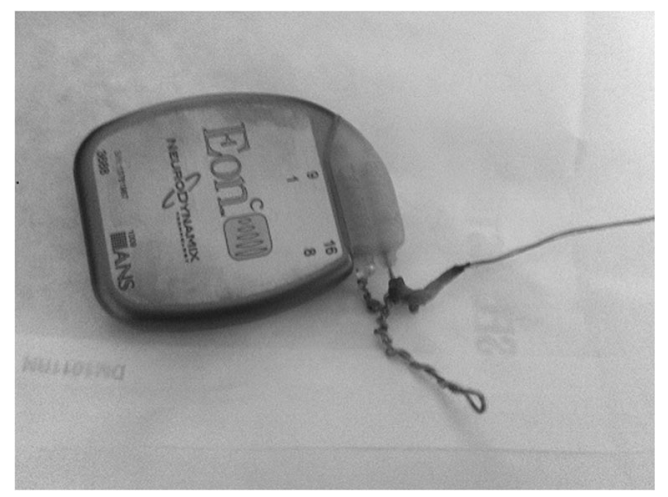

Fig. 3 IPG with twisted wires after removal from patient

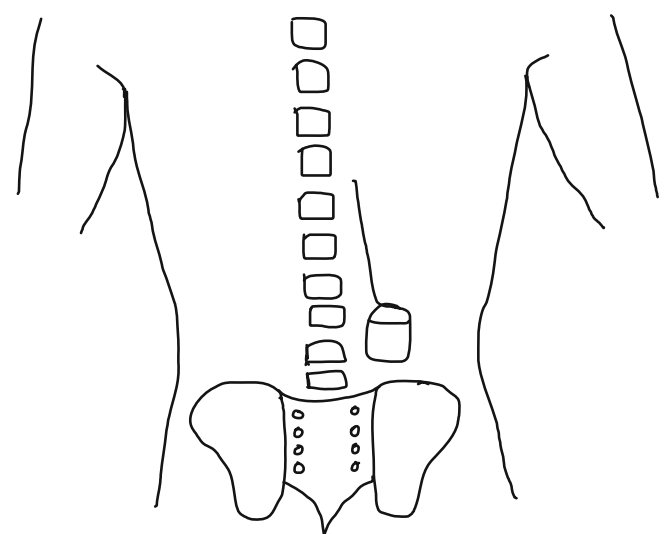

Fig. 4 Schematic diagram illustrating IPG site for revision cases

\section{Discussion}

To the best of our knowledge and following a literature research by two independent investigators, we present the first reported case series on Twiddler's syndrome in SCS. All patients were female, aged between 31 and 54 years and all patients had varying degrees of depression and anxiety. Their BMI ranged between 31.9 and 39.3, which falls into the obese category according to the WHO classification [37]. There was no pattern to the timing of presentation, which ranged from 4 to 228 weeks after implantation. Our patients underwent validated psychological testing, which consisted of the Becks Depression Inventory [6] and the Pain Anxiety Symptoms Scale [29] prior to treatment which revealed varying degrees of depression and fear of pain.

The first SCS was implanted by Shealy et al. in 1967 [38]. Hardware failure is not an uncommon complication with SCS and it is reported to occur most commonly within the first 2 years after implantation [25, 43]. In Twiddler's syndrome with other implantable devices, presentation is reported between 6 months and 3 years $[3,9,16,17,21$, 31, 34, 39].

Hardware-related complications in SCS have different aetiologies, including lead migration/fracture, electrical shorting-out with cessation of stimulation, infection, decreased stimulation and battery end of life. Such complications have been reported to be between 22 and $27.2 \%$ is SCS $[25,41]$. Hardware malfunction in Twiddler's syndrome is due to lead coiling, which can cause displacement and lead fracture $[9,16,20,34]$. Twisting of the wires in such a manner has not been reported with SCS previously $[8,23,26]$.

Twiddler's syndrome typically presents with loss of stimulation, resulting in a recurrence of symptoms [32, 34]. There may be pain along the course of the wires due to movement and traction of the wires. Although Twiddler's syndrome is a rare condition, there may be an element of under-reporting of Twiddler's syndrome due to the lack of awareness of this problem in SCS. 


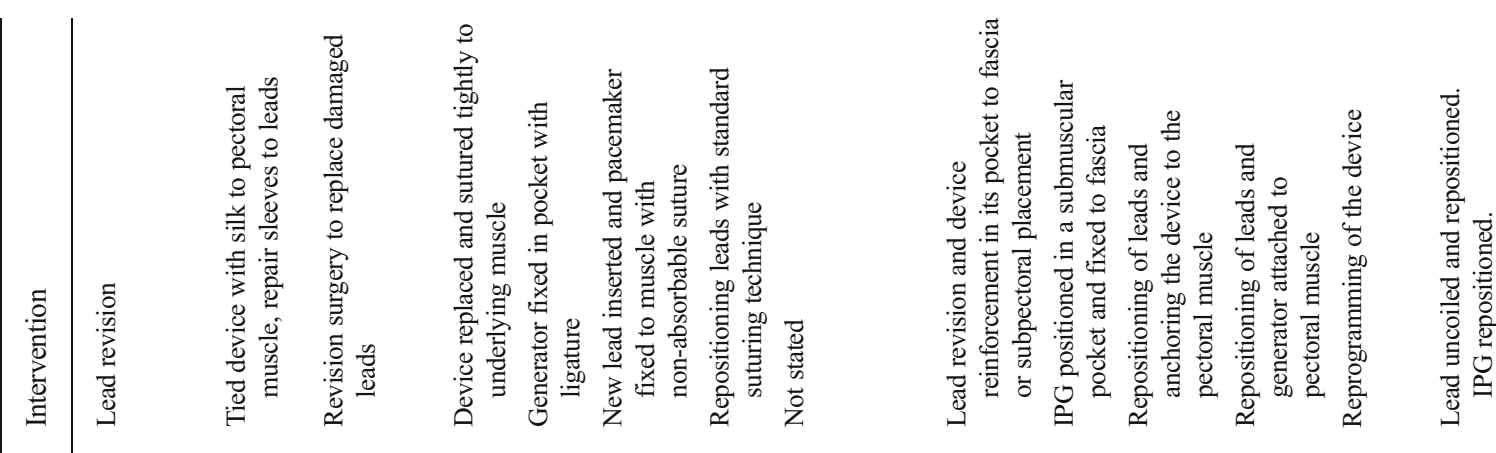

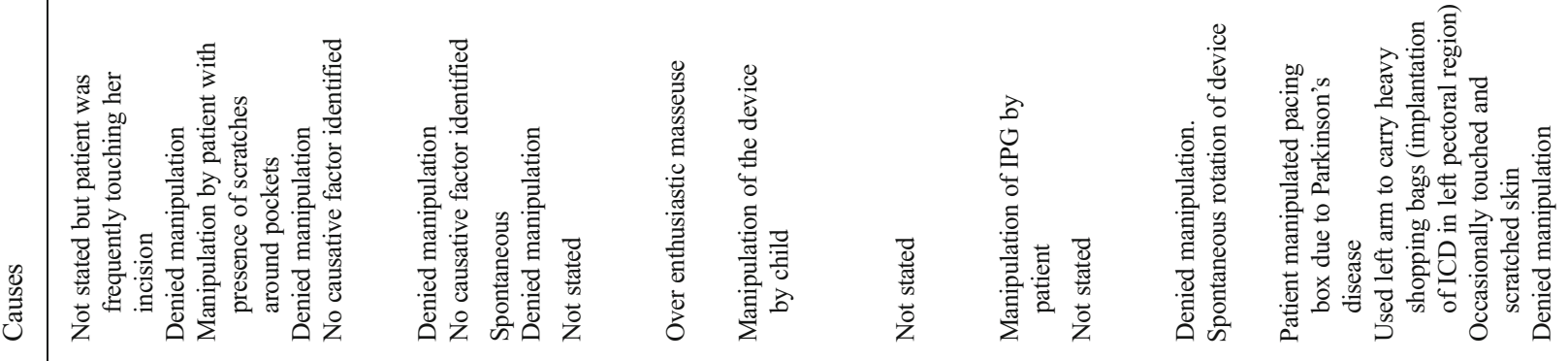

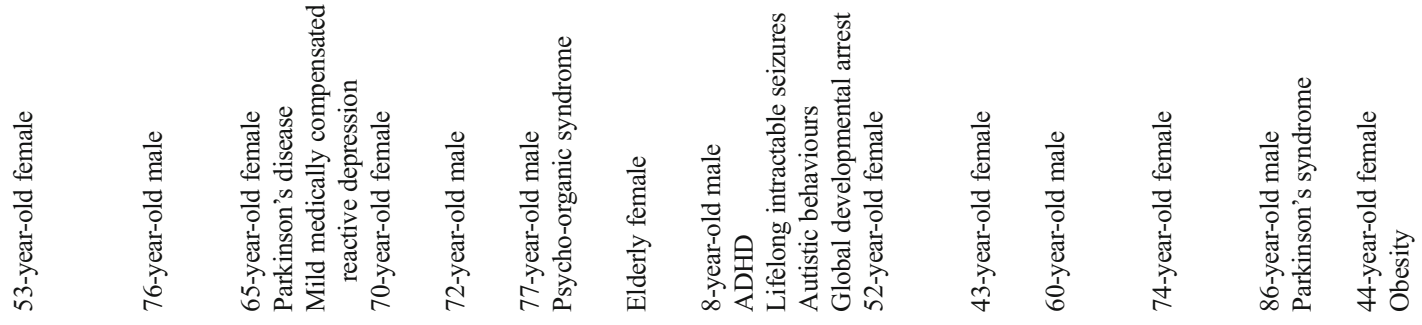
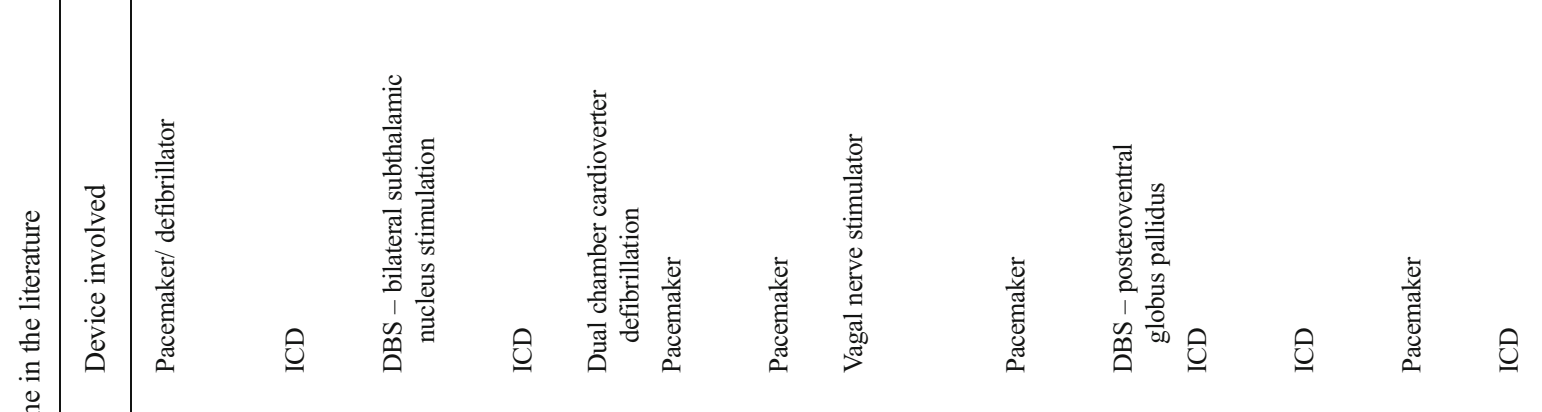

量

芯产

I

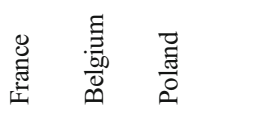

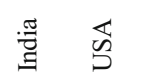

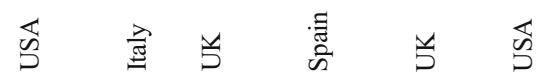

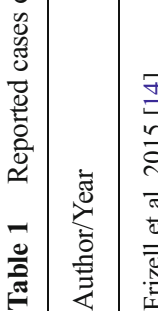

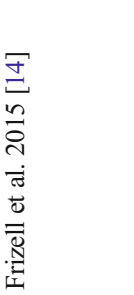

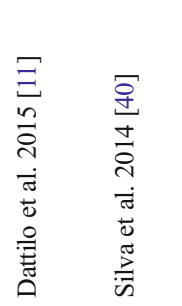

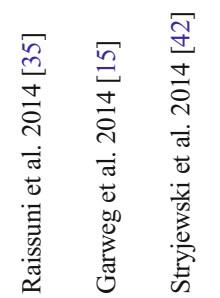

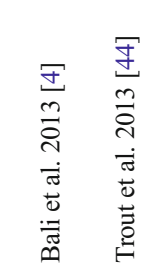

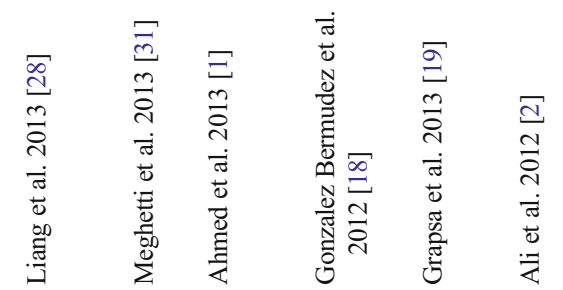




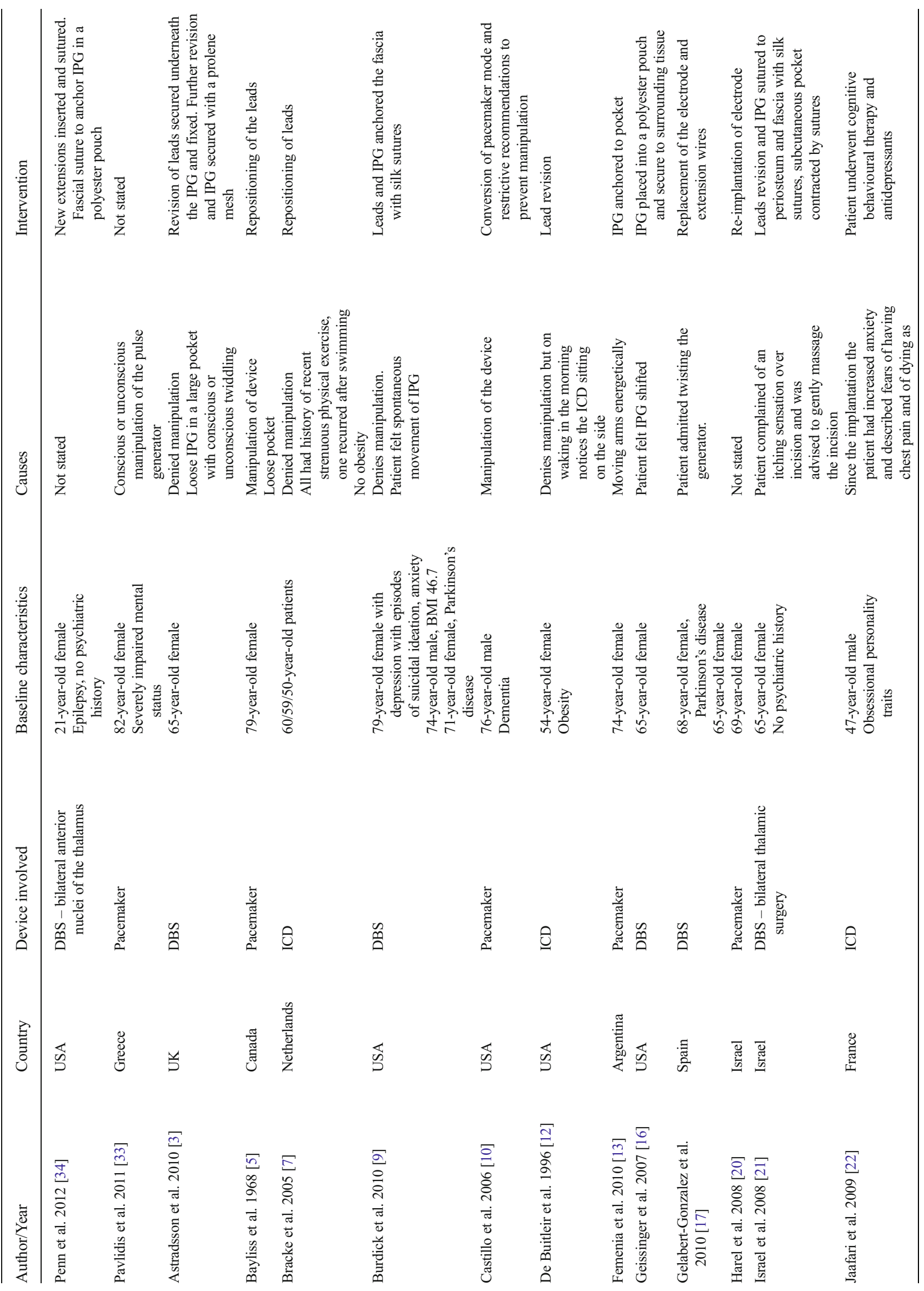


Twiddler's syndrome has most commonly been described with cardiac pacemakers and defibrillators $[5,7,12,13,19$, $24,27,28,30,32,39]$. More recently it has been described in deep brain stimulation $[3,9,16,17,21,31,34]$. Table 1 summarises all cases of Twiddler's syndrome reported in the literature with different implants. The aetiology of Twiddler's syndrome is not completely understood; however, some have found an association with obese women [7, 12, 16, 24, 34]. With increased subcutaneous tissue, rotation of the device may occur during normal activity, which in turn can lead to wire coiling on the IPG. In one case series [17], the patients admitted to actively twisting the IPG. However, in most reports patients deny manipulating the IPG. Physical activity has been postulated as a causative factor in IPGs implanted in the pectoral region $[7,16,34]$. An association has been noted with neurological and psychological conditions such as dementia, obsessive-compulsive disorders, seizures, depression and anxiety $[9,10,20,22,34]$. All patients in this series denied manipulating their IPGs. All our patients with Twiddler's had moderate to severe depression on preoperative psychological screening.

Reported measures to reduce the incidence of IPG displacement and Twiddler's syndrome include suture sleeves [27, 30]. Others have used prolene mesh, non-absorbable sutures, anchoring the IPG to the fascia, placing the IPG in a submuscular plane and limiting the pocket size to prevent further occurrences of Twiddler's syndrome [3, 17, 21, 31, 34]. We suggest that the relocation of the IPG to above the iliac crest allows the IPG to be anchored to the lumbar fascia. Twiddler's syndrome maybe less likely to occur with an IPG in this position as it is less accessible and visible to the patient. This approach has the added advantage of good access to the spine lead and IPG site simultaneously in the prone position. Another measure that may help prevent Twiddler's syndrome may be psychological intervention in those identified at risk of manipulating their IPGs [22].

\section{Conclusions}

To the best of our knowledge, this is the first reported series of Twiddler's syndrome with IPGs for spinal cord stimulation. There are many factors that may contribute to Twiddler's syndrome including the patient's weight, site of implantation and psychological disorders. Based on our case series, implanting the IPG in the lumbar region subcutaneously above the iliac crest may prevent recurrence of Twiddler's syndrome. This approach has the added advantage of easier access to the spine with tunnelling in the prone position. Psychological screening may aid in identifying those at risk of Twiddler's syndrome.

\section{Compliance with ethical standards}

Funding No funding was received for this research. 
Conflict of interest All authors certify that they have no affiliations with or involvement in any organization or entity with any financial interest (such as honoraria; educational grants; participation in speakers' bureaus; membership, employment, consultancies, stock ownership, or other equity interest; and expert testimony or patent-licensing arrangements), or non-financial interest (such as personal or professional relationships, affiliations, knowledge or beliefs) in the subject matter or materials discussed in this manuscript.

Ethical approval All procedures performed in studies involving human participants were in accordance with the ethical standards of the institutional and/or national research committee and with the 1964 Helsinki declaration and its later amendments or comparable ethical standards.

For this retrospective study formal consent is not required.

Informed consent Informed consent was obtained from all individual participants included in the study.

Open Access This article is distributed under the terms of the Creative Commons Attribution 4.0 International License (http:// creativecommons.org/licenses/by/4.0/), which permits unrestricted use, distribution, and reproduction in any medium, provided you give appropriate credit to the original author(s) and the source, provide a link to the Creative Commons license, and indicate if changes were made.

\section{References}

1. Ahmed FZ, Luckie M, Goode GK (2013) An unusual case of combined classic and reverse Twiddler's syndrome. Can J Cardiol 29: 1015.e1019-1015.e1010

2. Ali RG, Navaravong L, Cui J, Stoenescu M (2012) An uncommon twist on Twiddler's syndrome. Conn Med 76:81-83

3. Astradsson A, Schweder PM, Joint C, Green AL, Aziz TZ (2011) Twiddler's syndrome in a patient with a deep brain stimulation device for generalized dystonia. J Clin Neurosci 18:970-972

4. Bali HK, Chattree KK, Bali SK, Chauhan HK, Shukla CP (2013) A tale of early Reel syndrome caused by an over-enthusiastic masseuse. Indian Heart J 65:703-704

5. Bayliss CE, Beanlands DS, Baird RJ (1968) The pacemakerTwiddler's syndrome: a new complication of implantable transvenous pacemakers. CMAJ 99:371-373

6. Beck AT, Steer RA, Carbin MG (1988) Psychometric properties of the Beck Depression Inventory: twenty-five years of evaluation. Clin Psychol Rev 8:77-100

7. Bracke F, van Gelder B, Dijkman B, Meijer A (2005) Lead system causing Twiddler's syndrome in patients with an implantable cardioverter-defibrillator. J Thorac Cardiovasc Surg 129:231-232

8. Burchiel KJ, Anderson VC, Brown FD, Fessler RG, Friedman WA, Pelofsky S, Weiner RL, Oakley J, Shatin D (1996) Prospective, multicenter study of spinal cord stimulation for relief of chronic back and extremity pain. Spine 21:2786-2794

9. Burdick AP, Okun MS, Haq IU, Ward HE, Bova F, Jacobson CE, Bowers D, Zeilman P, Foote KD (2010) Prevalence of Twiddler's syndrome as a cause of deep brain stimulation hardware failure. Stereotact Funct Neurosurg 88:353-359

10. Castillo R, Cavusoglu E (2006) Twiddler's syndrome: an interesting cause of pacemaker failure. Cardiology 105:119-121

11. Dattilo G, Scarano M, Casale M, Sergi M, Quattrocchi S, Parato M, Imbalzano E (2015) An atypical manifestation of Twiddler syndrome. Int J Cardiol 186:1-2
12. de Buitleir M, Canver CC (1996) Twiddler's syndrome complicating a transvenous defibrillator lead system. Chest 109:1391-1394

13. Femenia F, Florentino C, Arrieta M, Arce M (2010) Iatrogenic Twiddler's syndrome: case report and proposed experimental model. Indian Pacing Electrophysiol J 10:517-521

14. Frizell AW, MacVane CZ (2015) Woman with syncope. Twiddler's syndrome. Ann Emerg Med 66(19):22

15. Garweg C, Alzand BS, Willems R (2014) Twiddler syndrome causing an inappropriate implantable cardioverter-defibrillator shock. Eur Heart J 35:516

16. Geissinger G, Neal JH (2007) Spontaneous Twiddler's syndrome in a patient with a deep brain stimulator. Surg Neurol 68:454-456, discussion 456

17. Gelabert-Gonzalez M, Relova-Quinteiro JL, Castro-Garcia A (2010) "Twiddler syndrome" in two patients with deep brain stimulation. Acta Neurochir (Wein) 152:489-491

18. Gonzalez Bermudez I, Pardo Fresno M, Garcia Campo E, Arnaiz Betolaza L, Crespo Carazo N, Beiras Torrado X (2012) Twiddler syndrome as a cause of defibrillator malfunction. Rev Port Cardiol 31:837-838

19. Grapsa J, Koa-Wing M, Fox KF (2013) Fiddling with the pacemaker: Twiddler's syndrome in a Parkinsonian patient. Perfusion 28:31-33

20. Harel G, Georgeta E, Copperman Y (2008) Twiddler's syndrome: a rare cause of pacemaker failure. Isr Med Assoc J 10:160-161

21. Israel Z, Spivak A (2008) A tremulous Twiddler. Stereotact Funct Neurosurg 86:297-299

22. Jaafari N, Bachollet MS, Paillot C, Amiel A, Rotge JY, Lafay N, Quentin S, Wassouf I, Camus V, Senon JL, El Hage W (2009) Obsessive compulsive disorder in a patient with Twiddler's syndrome. Pacing Clin Electrophysiol 32:399-402

23. Kemler MA, Barendse GA, van Kleef M, de Vet HC, Rijks CP, Furnee CA, van den Wildenberg FA (2000) Spinal cord stimulation in patients with chronic reflex sympathetic dystrophy. N Engl J Med 343:618-624

24. Khalilullah M, Khanna SK, Gupta U, Padmavati S (1979) Pacemaker Twiddler's syndrome: a note on its mechanism. J Cardiovasc Surg (Torino) 20:95-100

25. Kumar K, Buchser E, Linderoth B, Meglio M, Van Buyten JP (2007) Avoiding complications from spinal cord stimulation: practical recommendations from an international panel of experts. Neuromodulation 10:24-33

26. Kumar K, Taylor RS, Jacques L, Eldabe S, Meglio M, Molet J, Thomson S, O'Callaghan J, Eisenberg E, Milbouw G, Buchser E, Fortini G, Richardson J, North RB (2008) The effects of spinal cord stimulation in neuropathic pain are sustained: a 24-month follow-up of the prospective randomized controlled multicenter trial of the effectiveness of spinal cord stimulation. Neurosurgery 63:762 770, discussion 770

27. Lal RB, Avery RD (1990) Aggressive pacemaker Twiddler's syndrome. Dislodgement of an active fixation ventricular pacing electrode. Chest 97:756-757

28. Liang JJ, Fenstad ER (2013) Twiddler's syndrome. Lancet 382:e47

29. McCracken LM, Zayfert C, Gross RT (1992) The pain anxiety symptoms scale: development and validation of a scale to measure fear of pain. Pain 50:67-73

30. Mehta D, Lipsius M, Suri RS, Krol RB, Saksena S (1992) Twiddler's syndrome with the implantable cardioverter-defibrillator. Am Heart J 123:1079-1082

31. Menghetti C, Zekaj E, Saleh C, Porta M, Servello D (2013) How to avoid Twiddler's syndrome in deep brain stimulation for dystonia? Neuromodulation 17:198-199

32. Nicholson WJ, Tuohy KA, Tilkemeier P (2003) Twiddler's syndrome. N Engl J Med 348:1726-1727

33. Pavlidis AN, Orfanidis Z, Levantakis IP, Giannakopoulos A, Manolis AJ (2011) Twiddler's syndrome. Acute Card Care 13:194 
34. Penn DL, Wu C, Skidmore C, Sperling MR, Sharan AD (2012) Twiddler's syndrome in a patient with epilepsy treated with deep brain stimulation. Epilepsia 53:e119-e121

35. Raissuni Z, Douchet MP, Chauvin M (2014) Where is the lead? An uncommon twist in a defibrillator Twiddler syndrome. Kardiol Pol 72:204

36. Rosenow JM, Stanton-Hicks M, Rezai AR, Henderson JM (2006) Failure modes of spinal cord stimulation hardware. J Neurosurg Spine 5:183-190

37. Seidell JC, Flegal KM (1997) Assessing obesity: classification and epidemiology. Br Med Bull 53:238-252

38. Shealy CN, Mortimer JT, Reswick JB (1967) Electrical inhibition of pain by stimulation of the dorsal columns: preliminary clinical report. Anesth Analg 46:489-491

39. Sidhu GS, Seifi A, Zangiabadi AH, Reinig M (2009) Follow your leads: a case of Twiddler's syndrome. South Med J 102:871-872

40. Silva PA, Chamadoira C, Costa H, Linhares P, Rosas MJ, Vaz R (2014) Twiddler (or Not) syndrome: questioning etiology for an uncommon form of hardware malfunction in deep brain stimulation. Surg Neurol Int 5:S410-S412

41. Spincemaille GH, Klomp HM, Steyerberg EW, van Urk H, Habbema JD (2000) Technical data and complications of spinal cord stimulation: data from a randomized trial on critical limb ischemia. Stereotact Funct Neurosurg 74:63-72

42. Stryjewski PJ, Kuczaj A, Kulak L, Nowak J, Nessler B, Nessler J (2014) Twiddler's syndrome: a rare complication of pacemaker implantation. Pol Arch Med Wewn 124:209

43. Taylor RS, Van Buyten JP, Buchser E (2005) Spinal cord stimulation for chronic back and leg pain and failed back surgery syndrome: a systematic review and analysis of prognostic factors. Spine 30:152-160

44. Trout AT, Larson DB, Mangano FT, Gonsalves CH (2013) Twiddler syndrome with a twist: a cause of vagal nerve stimulator lead fracture. Pediatr Radiol 43:1647-1651 